\title{
Association between the incidence and mortality rates for corpus uteri cancer and human development index (HDI): a global ecological study
}

\author{
Zaher Khazaei, MSc ${ }^{1}$, Elham Goodarzi, MSc ${ }^{2}$, Malihe Sohrabivafa, PhDc ${ }^{3}$, Hasan Naemi, MSc ${ }^{4}$, \\ Kamyar Mansori, PhD ${ }^{5}$ \\ ${ }^{1}$ Department of Epidemiology, School of Public Health, Ilam University of Medical Sciences, Ilam; ${ }^{2}$ Department of Public Health, School of Public \\ Health, Social Determinants of Health Research Center, Lorestan University of Medical Sciences, Khorramabad; ${ }^{3}$ Department of Public Health, School \\ of Medicine, Dezful University of Medical Sciences, Dezful; ${ }^{4}$ Iranian Research Center on Healthy Aging, Sabzevar University of Medical Sciences, \\ Sabzevar; ${ }^{5}$ Department of Biostatistics and Epidemiology, School of Medicine, Zanjan University of Medical Sciences, Zanjan, Iran
}

\section{Objective}

The aim of this study was to determine the association between the incidence of and mortality due to corpus uteri cancer (CUC) and the human development index (HDI) across the world.

\section{Methods}

This was an ecological study. The incidence and mortality rates of CUC along with HDI data were extracted from the Global Cancer Data in 2018. Subsequently, correlation coefficient and linear regression model were used to determine the association between the incidence and mortality rates of CUC and the HDI. STATA-14 was used for data analysis.

Results

There was a positive and significant correlation between the incidence $(r=0.693 ; P<0.001)$ and mortality $(r=0.284$; $P<0.001)$ rates of uterine cancer and the HDI. A positive and significant correlation was also observed between the incidence rate and the gross national income per 1,000 capita $(r=0.440 ; P<0.001)$, mean years of schooling (MYS) $(r=0.740 ; P<0.001)$, life expectancy at birth (LEB) $(r=0.590 ; P<0.001)$, and expected years of schooling $(r=0.650 ; P<0.001)$. The results of the linear regression model showed a significant statistical association between MYS and the incidence of CUC $(\beta=1.10 ; 95 \%$ confidence interval $[\mathrm{Cl}], 0.60-1.70)$ and LEB and mortality due to uteri cancer $(\beta=0.40 ; 95 \% \mathrm{Cl}$, $0.10-0.90)$.

\section{Conclusion}

The results of this study suggest a significant statistical association between the incidence and mortality rates of CUC and the HDI.

Keywords: Incidence; Mortality; Uterine neoplasms; Human development index; World

\section{Introduction}

Endometrial cancer is the sixth most common cancer worldwide. Moreover, it is the most common female genital cancer in developed countries such as Europe, the United States, Russia, and Canada [1]. More than $90 \%$ of corpus uteri cancers (CUCS) are epithelial tumors. There are 2 types of endometrial cancers which can be differentiated according to clinical, tissue, and molecular characteristics. The type I is estrogen-dependent and accounts for $85 \%$ of all endometrial cancers, often occurs in the younger women and has a
Received: 2019.08.25. Revised: 2019.10.12. Accepted: 2019.10.28. Corresponding author: Kamyar Mansori, $\mathrm{PhD}$

Department of Biostatistics and Epidemiology, School of Medicine, Zanjan University of Medical Sciences, Zanjan, Iran

E-mail: kamyarmansori@yahoo.com

https://orcid.org/0000-0003-3527-4741

Articles published in Obstet Gynecol Sci are open-access, distributed under the terms of the Creative Commons Attribution Non-Commercial License (http://creativecommons. org/licenses/by-nc/3.0/) which permits unrestricted non-commercial use, distribution, and reproduction in any medium, provided the original work is properly cited.

Copyright $\odot 2020$ Korean Society of Obstetrics and Gynecology 


\title{
Obstetrics \& Gynecology Science
}

\author{
Vol. 63, No. 2, 2020
}

good prognosis. On the other hand, type II including nonendometriosis cancers occurs predominantly at age $\geq 70$ years and are characterized by lower survival rates [2].

Approximately 290,000 new cases of endometrial cancer occur annually worldwide. The incidence rate of this cancer is higher in high-income countries than in low-income countries (5.5\% vs. $4.2 \%)$. However, its mortality rate is higher in low-income countries. The cumulative risk of endometrial cancer by age 75 for high-income and low-income countries is $1.6 \%$ and $0.7 \%$; respectively [3]. In 2012, the highest incidence was reported for North America (19.1 per 100,000), and Western and Northern Europe (12.9-15.6 per 100,000). The lowest incidence rates were reported for South Asia (2.7 per 100,000) and Africa ( $<5$ per 100,000) [4]. In the United States, there were 49,560 new cases and 8,190 deaths due to endometrial cancer in 2013 [5].

The epidemic of obesity and physical inactivity are 2 important risk factors for endometrial cancer in high-income countries [6]. On the other hand, physical activity, prolonged use of estrogen and progesterone therapy are associated with a reduction in the risk of endometrial cancer $[7,8]$. Studies have shown that menstrual-related risk factors, childbirth at older ages, consumption of external hormone (contraceptive pills and hormone replacement therapy), nutrition (alcohol consumption) and anthropometrics (more weight, weight gain during adulthood and body fat distribution) are important risk factors related to the high incidence of this cancer in countries with a high human development index (HDI) $[9,10]$. In other words, HDI which reflects the social and economic status of people in different countries, may be associated with the incidence of endometrial cancer [11-13]. HDI is a function of health, quality of life, access to healthcare, lack of anxiety, relaxation, economic and social security. Studies have shown that the HDI can be an important factor for classification and comparison of cancer in different communities $[14,15]$. However, limited studies have examined the relationship between the HDI and CUC. Therefore, the aim of this study was to determine the association between the incidence and mortality rates of CUC and the HDI across the world.

\section{Materials and methods}

\section{Incidence rate}

The methods used to estimate the sex- and age-specific incidence rates of cancer in a specific country fall into the following broad categories, in order of priority: 1) Observed national incidence rates were projected to 2018 (45 countries). 2) The most recently observed incidence rates (national or regional) were applied to the 2018 population (50 countries). 3) Rates were estimated from national mortality data by modeling, using mortality-to-incidence ratios derived from cancer registries in that country (14 countries). 4) Rates were estimated from national mortality estimates by modeling, using mortality-to-incidence ratios derived from cancer registries in neighboring countries (37 countries). 5) Age- and sex-specific national incidence rates for all cancers combined were obtained by averaging the overall rates from neighboring countries. These rates were then partitioned to obtain the national incidence for specific sites using available cancer-specific relative frequency data (7 countries). 6) Rates were estimated as an average of those from selected neighboring countries (32 countries).

\section{Mortality rate}

The methods used to estimate the sex- and age-specific mortality rates of cancer in a specific country fall into the following broad categories, in order of priority: 1) Observed national mortality rates were projected to 2018 (81 countries). 2) The most recently observed national mortality rates were applied to the 2018 population (20 countries). 3) Rates were estimated from the corresponding national incidence estimates by modeling, using incidence-to-mortality ratios derived from cancer registries in neighboring countries (81 countries). 4) Rates were estimated as an average of those from selected neighboring countries (3 countries) [16-18].

\section{Human development index}

The HDI provided by the GLOBOCAN provides the latest data on global development, which includes national, regional, and global estimates. According to the Human Development Report, countries are divided into 4 groups of very high human development, high human development, medium human development, and low human development based on the level of the HDI. The numerical value of the HDI is between 0 and 1. It represents the efforts taken by each country to reach the highest possible HDI value and also allows comparisons between countries. HDI represents diverse human development measures. It measures the mean success of a country in 3 main dimensions of human development: 


\section{Obstetrics \& Gynecology Science}

Zaher Khazaei, et al. Corpus uteri cancer and HDI

a long and healthy life, access to education and proper living standards. The different dimensions of HDI include the following: 1) Mean years of schooling (MYS): average number of completed years of education of a population (HDI: 25 years and older). 2) Gross national income (GNI) per capita: the sum of value added by all resident producers plus any product taxes (less subsidies) not included in the valuation of output plus net receipts of primary income (compensation of employees and property income) from abroad. 3) Expected years of schooling (EYS): the number of years during which a 2-year-old child can expect to spend in schooling, based on the school enrolment rates at a given date. 4) Life expectancy at birth (LEB): the number of years a newborn infant could expect to live if prevailing patterns of age-specific mortality rates at the time of birth stay the same throughout the infant's life [19-22].

\section{Ethical considerations}

This study is based on the Global Cancer Data Record, and incidence and mortality data are generally reported for each country.

\section{Statistical analysis}

In descriptive analysis, mean (standard deviation) and frequency (\%) were reported for quantitative and qualitative variables, respectively. In this study, correlation coefficient and multiple linear regression model were used to determine the association between the incidence and mortality rates of CUC and the HDI and its dimensions (GNI per capita, MYS,

A Estimated number of deaths in 2018, corpus uteri, all ages

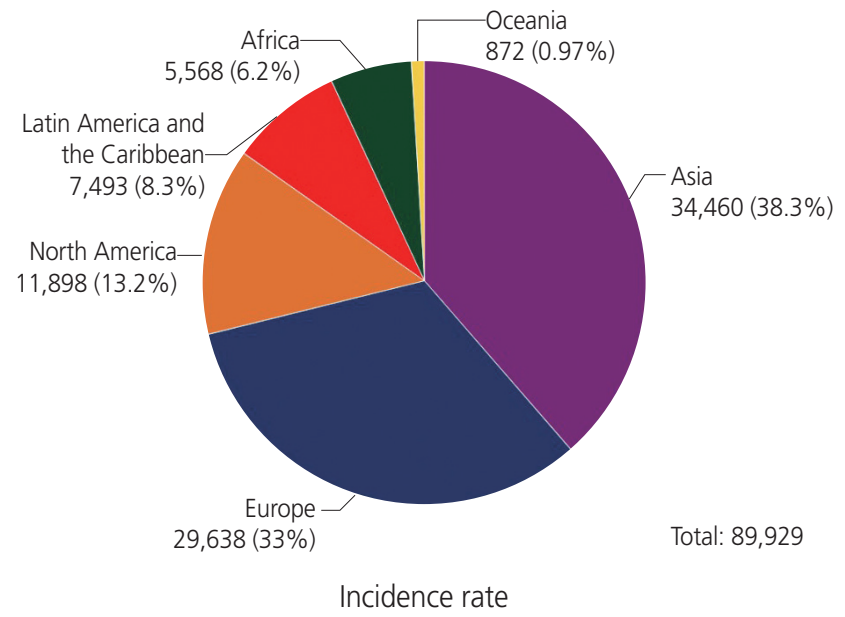

LEB, and EYS). Stata-14 software (StataCorp, College Station, TX, USA) was used for data analysis. Statistical significance was set at $P<0.05$.

\section{Results}

Based on 2018 records, 8,622,539 new cancer cases and $4,169,387$ deaths due to cancer were recorded in women of which 382,069 were new cases (4.4\%) and 89,929 (1.10\%) deaths were due to CUC. The highest number of new CUC cases $(148,764$ cases, $38.9 \%)$ and the highest number of deaths resulting from it (34,460 cases, 38.3\%) was reported for Asia (Fig. 1).

Table 1 shows the incidence and mortality rate of CUC in each continent. The results of our study demonstrated that the highest incidence of CUC in the world was in Be-

Table 1. Corpus uteri cancer incidence and mortality rates in different human development Index (HDI) regions in 2018

\begin{tabular}{lcccccc}
\hline \multirow{2}{*}{ HDI } & \multicolumn{2}{c}{ Incidence rate } & & \multicolumn{2}{c}{ Mortality rate } \\
\cline { 2 - 3 } \cline { 5 - 6 } & CR & ASR & & CR & ASR \\
\hline Very high & 26.5 & 14.5 & & 6.1 & 2.6 \\
High & 13.0 & 9.4 & & 3.7 & 2.4 \\
Medium & 3.7 & 4.4 & & 1.1 & 1.5 \\
Low & 1.9 & 3.4 & & 0.9 & 1.8 \\
$P$-value (F-test) & $<0.001$ & $<0.001$ & & $<0.001$ & $<0.001$ \\
\hline
\end{tabular}

$\mathrm{CR}$, crude rate; ASR, age-standardized rate.

B Estimated number of new cases in 2018, corpus uteri, all ages

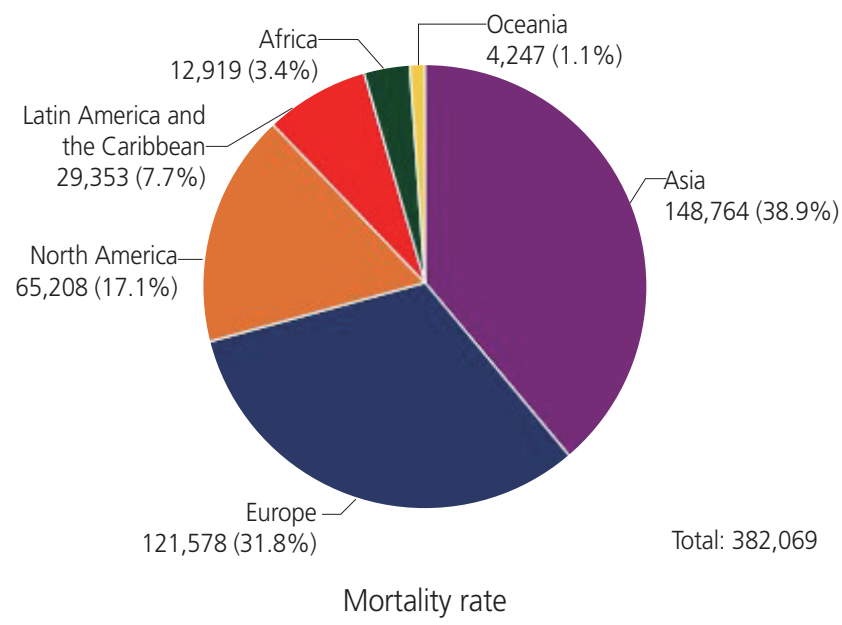

Fig. 1. Incidence (A) and mortality rates (B) of corpus uteri cancer in females in 2018, by continents [17]. 


\section{Obstetrics \& Gynecology Science}

Vol. 63, No. 2, 2020

larus $(24.9 \%$ in 100,000$)$ and Samoa $(24.8 \%$ in 100,000$)$. The highest incidence of CUC mortality in Samoa $(4.6 \%$ 100,000) and Trinidad and Tobago (6.2 in 100,000) (Fig. 2). Based on the 2018 cancer report, the highest incidence (7.15 per 100,000$)$ and mortality $(6.2$ per 100,000$)$ of the CUC was found in the very high $\mathrm{HDI}$ regions (Fig. 3).

The variance analysis showed that the highest mean incidence $(14.5$ out of 100,000$)$ was found in areas with very high human development, and the lowest incidence (3.4 out of 100,000 ) was observed in areas of low human de- velopment, and the difference was statistically significant $(P<0.001)$. The highest rate of mortality $(2.6$ per 100,000$)$ was noted for areas with very high human development, and the lowest mortality rate $(1.5$ per 100,000$)$ was observed for areas with medium human development and the difference was statistically significant $(P<0.001)$ (Table 1).

The results showed that there was a positive and significant correlation between the incidence $(r=0.693 ; P<0.001)$ and mortality $(r=0.284 ; P<0.001)$ rates of CUC and the HDI (Fig. 4). The results showed that there was a positive and

A Incidence rate
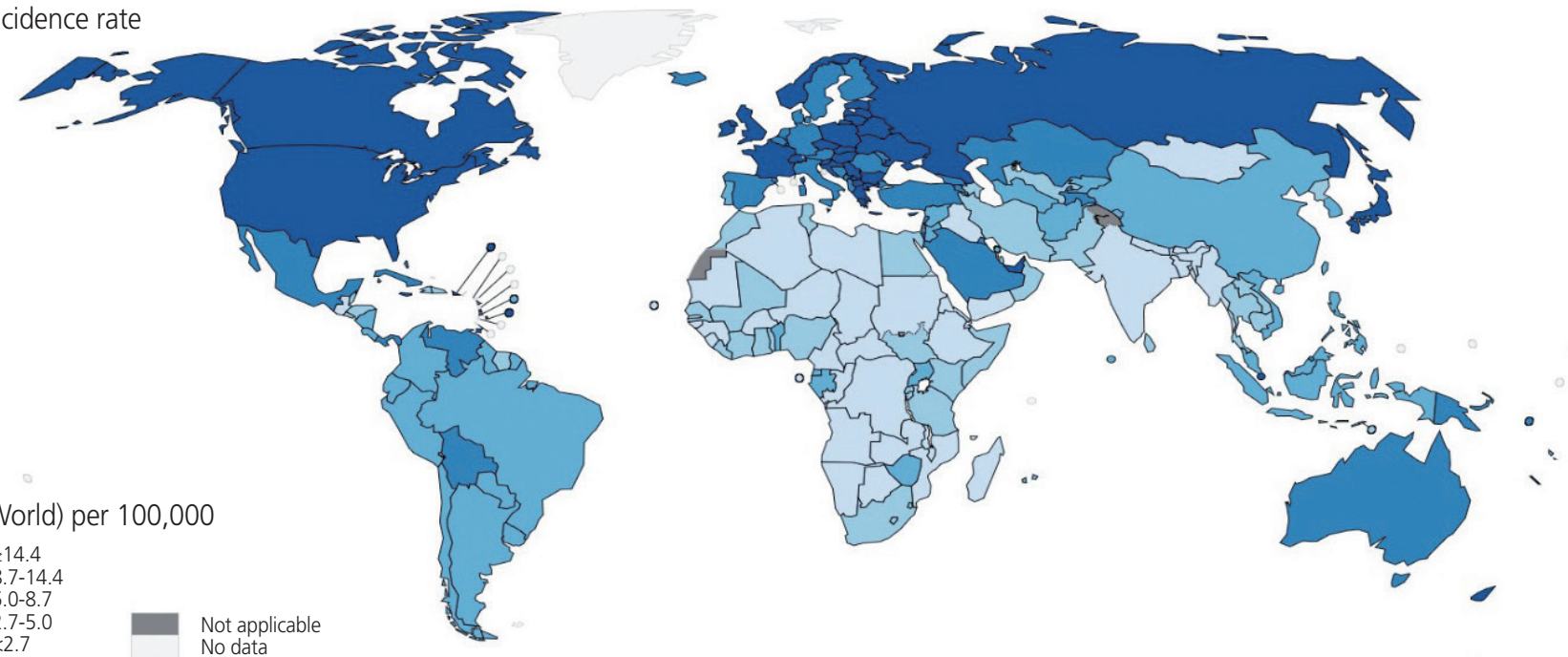

B Mortality rate
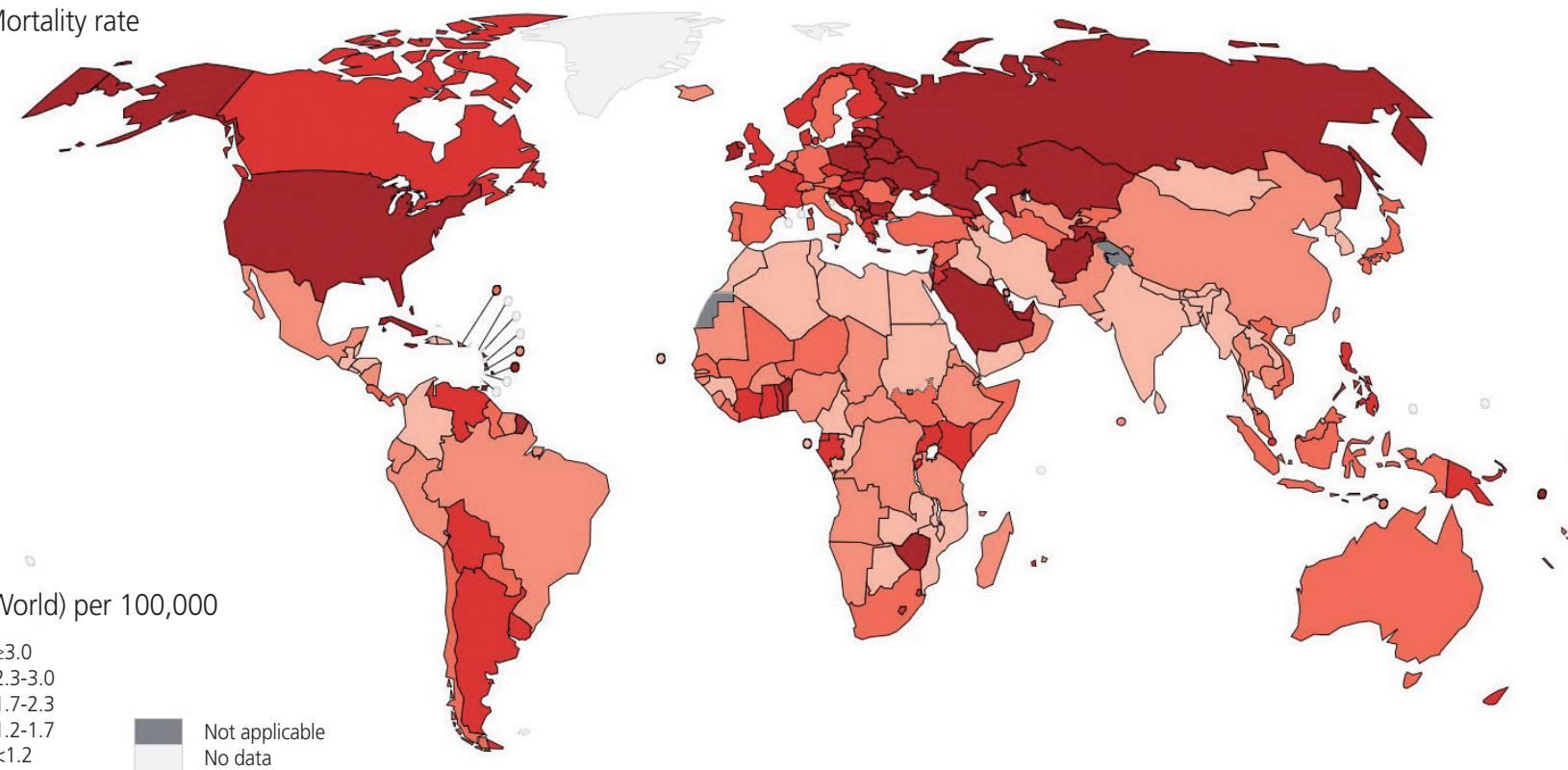

Fig. 2. Estimated age-standardized incidence and mortality rates for corpus uteri cancer across the world in 2018 [17]. ASR, age-standardized rate. 


\section{Obstetrics \& Gynecology Science}

Zaher Khazaei, et al. Corpus uteri cancer and HDI

significant correlation between the incidence rate and $\mathrm{GNI}$ $(r=0.44 ; P<0.001)$, MYS ( $r=0.74 ; P<0.001), L E B(r=0.59$; $P<0.001)$ and EYS $(r=0.65 ; P<0.001)$. There was a positive and significant correlation between the incidence rate and GNI ( $r=0.16 ; P<0.001)$, MYS $(r=0.42 ; P<0.001)$, LEB $(r=0.25$; $P<0.001)$ and $\mathrm{EYS}(\mathrm{r}=0.65 ; P<0.001)$ (Table 2).

The linear regression model showed that increased MYS ( $\beta=1.1 ; 95 \%$ confidence interval $[\mathrm{Cl}], 0.6-1.7$ ) significantly decreased the incidence of the CUC $(P<0.05)$. According to the regression analysis, the increase in MYS $(\beta=0.2 ; 95 \%$ $\mathrm{Cl}, 0.1-0.4)$ and LEB ( $\beta=0.4 ; 95 \% \mathrm{Cl}, 0.1-0.9)$ significantly increased mortality due to CUC $(P<0.05)$ (Table 3$)$.

\section{Discussion}

According to the results of the present study, the highest incidence $(15.7$ per 100,000) and mortality $(2.6$ per 100,000) of CUC were in areas with very high $\mathrm{HDI}$, and a positive and significant correlation between the incidence $(r=0.693$; $P<0.001)$ and mortality $(r=0.284 ; P<0.001)$ rates of CUC and $\mathrm{HDI}$ were observed.

The results of the HDI decomposition showed a significant positive correlation between the incidence and mortality rates and GNI, MYS, LEB, and EYS indices. In the developed world, CUC is the most common female genital malignancy as well as the most common cancer among American

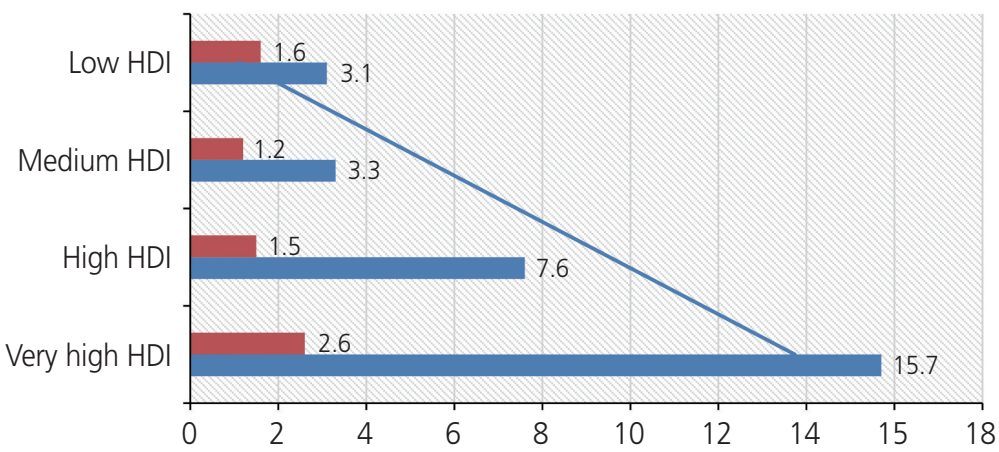

\begin{tabular}{|c|c|c|c|c|}
\cline { 2 - 5 } \multicolumn{1}{c|}{} & Very high HDI & High HDI & Medium HDI & Low HDI \\
\hline Mortality & 2.6 & 1.5 & 1.2 & 1.6 \\
\hline ancidence & 15.7 & 7.6 & 3.3 & 3.1 \\
\hline
\end{tabular}

Fig. 3. Distribution of incidence and mortality rates for corpus uteri cancer by human development index (HDI).

A R Sq. linear $=0.693, P<0.0001$

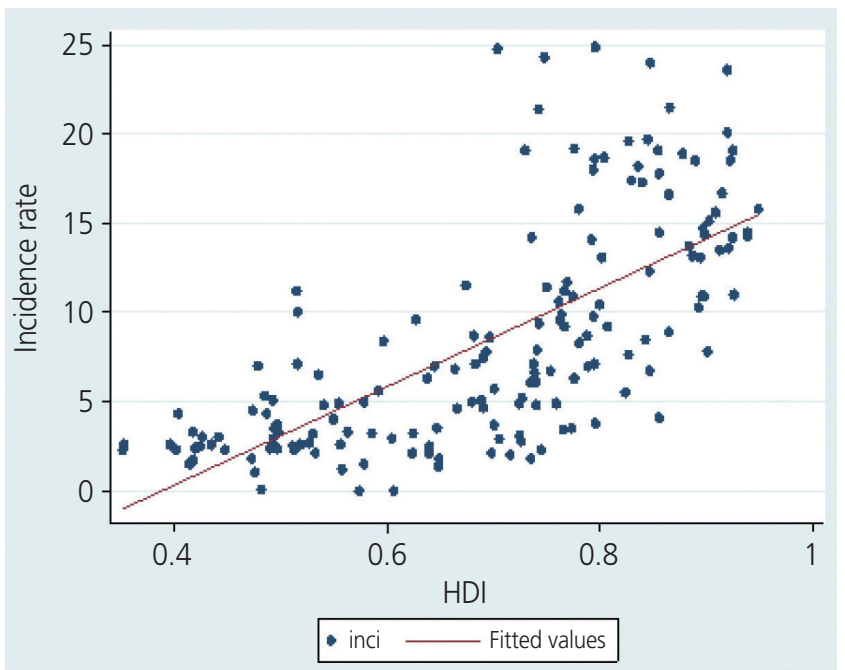

B R Sq. linear $=0.284, P<0.0001$

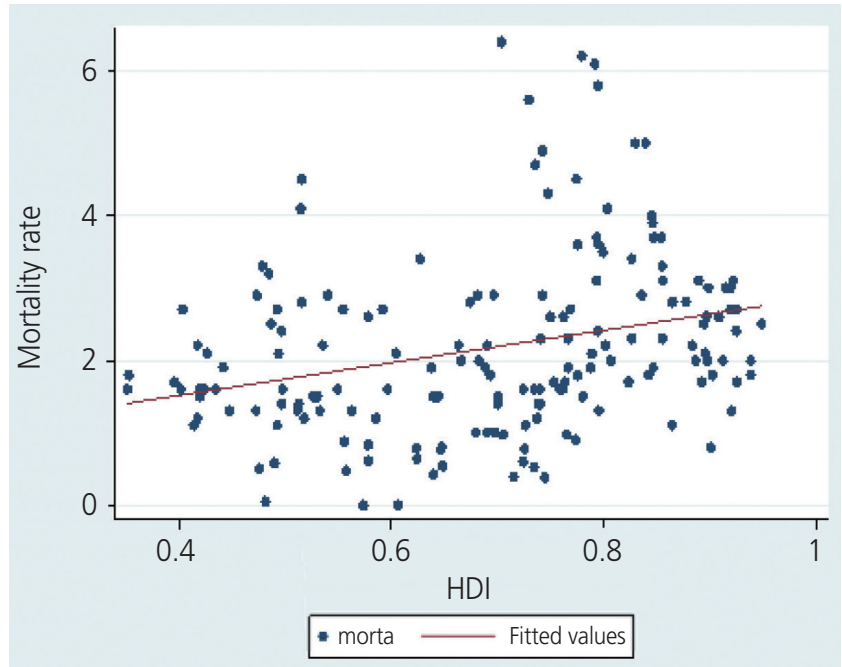

Fig. 4. Correlation between the human development index ( $\mathrm{HDI}$ ) and the incidence (A) and mortality rates (B) of corpus uteri cancer across the world in 2018. 


\title{
Obstetrics \& Gynecology Science
}

\author{
Vol. 63 , No. 2, 2020
}

women $[23,24]$. In western countries, it accounts for $9 \%$ to $6 \%$ of all cancers in women. In North America and Europe, endometrial cancer is the most common female genital cancer and the fourth most common cancer after breast, lung, and colorectal cancers. Several factors contribute to CUC. Some of the risk factors are connected with lifestyle changes in many countries [25]. The results of our study showed that the highest incidence $(15.7 \%$ in 100,000$)$ and mortality $(2.6$ in 100,000) of CUC are in the areas with very high $\mathrm{HDI}$. There was a positive and significant correlation between the incidence $(r=0.693 ; P<0.001)$ and mortality $(r=0.284$; $P<0.001)$ rates of CUC and the HDI. Some cancers are positively correlated with the level of socioeconomic development [19].

In 2012, 13,1819 (6 per 100,000) new cases of cancers and 34,247 (1.5 per 100,000) deaths were due to CUC. The highest incidence of CUC in Asia was in Armenia (26.7 per $100,000)$ and Israel $(15.4$ per 100,000$)$ in the age group of 60-64 years, respectively. Moreover, the highest death rate from CUC was in Armenia (1.6 in 100,000) and Afghanistan $(1.4$ in 100,000) [26]. Another study reported that 23,700 women who died in Europe in 2012 had endometrial cancer, making it the eighth cause of death from cancers in women
$[3,27]$. The incidence among white women is slightly higher compared to black women. However, the mortality rate among black women is almost twice as high compared to other groups of women [28]. An increase in the incidence of endometrial cancer after 2002 may be due to the widespread erosion of estrogen. On the other hand, the use of progesterone meningococcal hormonal therapy reduces the risk of endometrial cancer in women [29].

The differences in the incidence and mortality of cervical cancer in different countries may be due to differences in the incidence of human papillomavirus (HPV), cervical cancer screening, and treatment rate of the cases. Since the introduction of prevention programs, in particular pap smear, vaccination against HPV and initial treatment, the incidence and mortality of cervical cancer has declined steadily [30].

The analyses of cohort and case studies also support the positive relationship between overweight and obesity and the risk of CUC among both Asians and non-Asians [25,31]. Due to the strong association between endometrial cancer and body weight and its prevalence in the United States, Central and Eastern Europe and several other European countries (Norway, England, Spain), obesity in these areas may be considered a risk factor for endometrial cancer [32].

Table 2. Pearson correlation between the human development index (HDI) components and ASIR and ASMR

\begin{tabular}{|c|c|c|c|c|}
\hline \multirow{2}{*}{ HDI components } & \multicolumn{2}{|c|}{ ASIR } & \multicolumn{2}{|c|}{ ASMR } \\
\hline & $r$ & $P$-value & $r$ & $P$-value \\
\hline Gross national income per 1,000 capita & 0.44 & $<0.001$ & 0.16 & $<0.001$ \\
\hline Mean years of schooling & 0.74 & $<0.001$ & 0.42 & $<0.001$ \\
\hline Life expectancy at birth & 0.59 & $<0.001$ & 0.25 & $<0.001$ \\
\hline Expected years of schooling & 0.65 & $<0.001$ & 0.65 & $<0.001$ \\
\hline
\end{tabular}

ASIR, age-standardized incidence rate; ASMR, age-standardized mortality rate.

Table 3. Determination of the association of human development index (HDI) and its components with the global incidence and mortality rates of corpus uteri cancer by multiple linear regression model (2018)

\begin{tabular}{|c|c|c|c|c|}
\hline \multirow{2}{*}{ Variables } & \multicolumn{2}{|c|}{ Incidence rate } & \multicolumn{2}{|c|}{ Mortality rate } \\
\hline & $\beta$-coefficient & $95 \% \mathrm{Cl}$ & $\beta$-coefficient & $95 \% \mathrm{Cl}$ \\
\hline $\mathrm{HDI}$ & 3.3 & $-18.60-25.40$ & 0.8 & $-4.80-6.50$ \\
\hline Gross national income per 1,000 capita & -0.08 & $-8.20-3.60$ & -0.03 & $-7.90-4.10$ \\
\hline Mean years of schooling & 1.1 & $0.60-1.70$ & 0.2 & $0.10-0.40$ \\
\hline Life expectancy at birth & 0.01 & $-0.10-0.20$ & 0.40 & $0.10-0.90$ \\
\hline Expected years of schooling & 0.1 & $-0.40-0.70$ & 0.07 & $-0.20-0.07$ \\
\hline
\end{tabular}

$\mathrm{Cl}$, confidence interval. 


\section{Obstetrics \& Gynecology Science}

Zaher Khazaei, et al. Corpus uteri cancer and HDI

Overweight is responsible for about $30 \%$ of cases of CUCs in 30 European countries [33]. The increasing prevalence of obesity is expected to be associated with an increase in the incidence of CUC in the coming years [34]. Researchers believe that many factors contribute to the development of cancers, which in 90-95\% of cases are due to environmental and lifestyle factors. The importance of $\mathrm{HDI}$ in the incidence and mortality of cancers has been confirmed in numerous studies [35]. The incidence of endometrial cancer increases with an increase in life expectancy [27].

Our results showed that there was a positive and significant correlation between the incidence rate of CUC and GNI $(r=0.44 ; P<0.001)$, MYS ( $r=0.74 ; P<0.001)$, LEB $(r=0.59$; $P<0.001)$ and EYS $(r=0.65 ; P<0.001)$. There was a significant positive correlation between the mortality rate and $\mathrm{GNI}$ $(r=0.16 ; P<0.001)$, MYS $(r=0.42 ; P<0.001), \operatorname{LEB}(r=0.25$; $P<0.001)$ and EYS $(r=0.65)$.

In the present study, an increase in $\mathrm{HDI}, \mathrm{GNI}$, and average years of education correlated with increased cancer morbidity and mortality. Accurate recording systems for cancers, more accurate screening tests, familiarity of women in society with signs of the cancer, and the detection of cancer cases in the early stages may have contributed to the findings in this study. Moreover, considering the impact of a number of socioeconomic and cultural factors on the development of CUC in different societies, it is necessary to raise the attention of women to their health, improve their awareness about the fatality of the disease and encourage them to participate in cancer screening programs.

In conclusion, the results of this study showed that the incidence and mortality of CUC are high in the countries with high HDI. Hence, considering the related risk factors in these countries could play an important role in reducing the incidence and mortality of this cancer. On the other hand, the high incidence and mortality of this cancer in countries with low HDI to countries with high HDI may be due to the lack of screening programs. This emphasizes the importance of screening programs in these countries to improve case detection and treatment of the disease towards reducing mortality. Although we observed a positive correlation between the incidence and mortality of CUC and HDI, other factors not captured in the HDI, such as life expectancy, security disparity, and certainly quality of health care provided, may equally affect cancer incidence and mortality and should be considered in the interpretation of results.

\section{Acknowledgements}

This paper used data from the GLOBOCAN and the United Nations Development Programme (UNDP), the authors thank them for their cooperation. The authors thank them for their cooperation. This study was supported by Kerman University of Medical Sciences, Kerman, Iran.

\section{Conflict of interest}

No potential conflict of interest relevant to this article was reported.

\section{Ethical approval}

This article emanates from a project approved by the Kerman University of Medical Sciences under the code of ethics IR.KMU.REC.1398.276.

\section{References}

1. Alghamdi IG, Hussain II, Alghamdi MS, El-Sheemy MA. The incidence rate of corpus uteri cancer among females in Saudi Arabia: an observational descriptive epidemiological analysis of data from Saudi Cancer Registry 2001-2008. Int J Womens Health 2014;6:141-7.

2. Santos P, Cunha TM. Uterine sarcomas: clinical presentation and MRI features. Diagn Interv Radiol 2015;21:4-9.

3. Siegel RL, Miller KD, Jemal A. Cancer statistics, 2015. CA Cancer J Clin 2015;65:5-29.

4. Ferlay J, Soerjomataram I, Dikshit R, Eser S, Mathers C, Rebelo $\mathrm{M}$, et al. Cancer incidence and mortality worldwide: sources, methods and major patterns in GLOBOCAN 2012. Int J Cancer 2015;136:E359-86.

5. American Cancer Society. Cancer facts \& figures 2013. Atlanta (GA): American Cancer Society; 2013.

6. Mansori K, Khateri S, Moradi Y, Khazaei Z, Mirzaei H, Hanis SM, et al. Prevalence of obesity and overweight in Iranian children aged less than 5 years: a systematic review and meta-analysis. Korean J Pediatr 2019;62:20612.

7. Friedenreich CM, Neilson HK, Lynch BM. State of the 


\section{Obstetrics \& Gynecology Science}

Vol. 63, No. 2, 2020

epidemiological evidence on physical activity and cancer prevention. Eur J Cancer 2010;46:2593-604.

8. Cust AE. Physical activity and gynecologic cancer prevention. Recent Results Cancer Res 2011;186:159-85.

9. Bray F, Ferlay J, Soerjomataram I, Siegel RL, Torre LA, Jemal A. Global cancer statistics 2018: GLOBOCAN estimates of incidence and mortality worldwide for 36 cancers in 185 countries. CA Cancer J Clin 2018;68:394424.

10. Chaichian S, Khateri S, Moradi Y, Shadmani FK, Mansori $\mathrm{K}$, Khazaei Z, et al. Trends in cervical cancer incidence in Iran from 2003 to 2009. Middle East J Cancer 2017:9:57-63.

11. Torres-Cintrón M, Ortiz AP, Ortiz-Ortiz KJ, FigueroaVallés NR, Pérez-Irizarry J, Díaz-Medina G, et al. Using a socioeconomic position index to assess disparities in cancer incidence and mortality, Puerto Rico, 1995-2004. Prev Chronic Dis 2012;9:E15.

12. Ghoncheh M, Mohammadian-Hafshejani A, Salehiniya H. Incidence and mortality of breast cancer and their relationship to development in Asia. Asian Pac J Cancer Prev 2015;16:6081-7.

13. Goodarzi E, Moslem A, Feizhadad H, Jarrahi AM, Adineh $H A$, Sohrabivafa $M$, et al. Epidemiology, incidence and mortality of thyroid cancer and their relationship with the human development index in the world: an ecology study in 2018. Adv Hum Biol 2019;9:162-7.

14. Trinh QD, Schmitges J, Sun M, Sammon J, Shariat $\mathrm{SF}$, Zorn $\mathrm{K}$, et al. Morbidity and mortality of radical prostatectomy differs by insurance status. Cancer 2012;118:1803-10.

15. Davoodi M, Bahadoram S, Bahadoram M, Barahman M, Khazaei Z, Amiri M. Impact of cancers on the kidney function and structure; an ignored entity. J Renal Inj Prev 2018;7:112-5.

16. Goodarzi E, Ghorat F, Mosavi Jarrahi A, Adineh HA, Sohrabivafa M, Khazaei Z. Global incidence and mortality of liver cancers and its relationship with the human development index (HDI): an ecology study in 2018. World Cancer Res J 2019;6:e1255.

17. International Agency for Research on Cancer. GLOBOCAN 2018. Lyon: International Agency for Research on Cancer; 2018 [cited 2018 Jan 17]. Available from: http:// gco.iarc.fr/today/datasources-methods.

18. Khazaei Z, Ghorat F, Jarrahi AM, Adineh HA, Sohrabiva- fa M, Goodarzi E. Global incidence and mortality of skin cancer by histological subtype and its relationship with the human development index (HDI); an ecology study in 2018. World Cancer Res J 2019;6:e1265.

19. Bray F, Jemal A, Grey N, Ferlay J, Forman D. Global cancer transitions according to the Human Development Index (2008-2030): a population-based study. Lancet Oncol 2012;13:790-801.

20. Khazaei S, Rezaeian S, Khazaei Z, Molaeipoor L, Nematollahi S, Lak P, et al. National Breast cancer mortality and incidence rates according to the human development index: an ecological study. Adv Breast Cancer Res 2016;5:30-6.

21. United Nations Development Programme. Human Development Report 2016. New York (NY): United Nations Development Programme; 2016 [cited 2018 Jan 17]. Available from: http://www.hdr.undp.org/en.

22. Khazaei Z, Mosavi Jarrahi A, Momenabadi V, Ghorat F, Adineh HA, Sohrabivafa $M$, et al. Global cancer statistics 2018: GLOBOCAN estimates of incidence and mortality worldwide stomach cancers and their relationship with the human development index (HDI). World Cancer Res J 2019;6:e1257.

23. Banas T, Juszczyk G, Pitynski K, Nieweglowska D, Ludwin A, Czerw A. Incidence and mortality rates in breast, corpus uteri, and ovarian cancers in Poland (1980-2013): an analysis of population-based data in relation to socioeconomic changes. Onco Targets Ther 2016;9:5521-30.

24. Sankaranarayanan R, Ferlay J. Worldwide burden of gynaecological cancer: the size of the problem. Best Pract Res Clin Obstet Gynaecol 2006;20:207-25.

25. Zhang Y, Liu H, Yang S, Zhang J, Qian L, Chen X. Overweight, obesity and endometrial cancer risk: results from a systematic review and meta-analysis. Int J Biol Markers 2014;29:e21-9.

26. Khazaei Z, Hasanpour Dehkordi A, Amiri M, Adineh HA, Sohrabivafa M, Darvishi I, et al. The incidence and mortality of endometrial cancer and its association with body mass index and human development index in Asian population. World Cancer Res J 2018;5:e1174.

27. Ferlay J, Steliarova-Foucher E, Lortet-Tieulent J, Rosso S, Coebergh JW, Comber $\mathrm{H}$, et al. Cancer incidence and mortality patterns in Europe: estimates for 40 countries in 2012. Eur J Cancer 2013;49:1374-403.

28. Jamison PM, Noone AM, Ries LA, Lee NC, Edwards BK. 


\section{Obstetrics \& Gynecology Science}

Zaher Khazaei, et al. Corpus uteri cancer and HDI

Trends in endometrial cancer incidence by race and histology with a correction for the prevalence of hysterectomy, SEER 1992 to 2008. Cancer Epidemiol Biomarkers Prev 2013;22:233-41.

29. Wartko P, Sherman ME, Yang HP, Felix AS, Brinton LA, Trabert B. Recent changes in endometrial cancer trends among menopausal-age U.S. women. Cancer Epidemiol 2013;37:374-7.

30. Kuehn B. Uterine Cancer Concern. JAMA 2019;321:336.

31. Lindemann K, Vatten LJ, Ellstrøm-Engh M, Eskild A. Body mass, diabetes and smoking, and endometrial cancer risk: a follow-up study. Br J Cancer 2008;98:1582-5.

32. Dossus L, Allen N, Kaaks R, Bakken K, Lund E, Tjonneland $A$, et al. Reproductive risk factors and endome- trial cancer: the European prospective investigation into cancer and nutrition. Int J Cancer 2010;127:442-51.

33. Renehan AG, Soerjomataram I, Tyson $M$, Egger $M$, Zwahlen M, Coebergh JW, et al. Incident cancer burden attributable to excess body mass index in 30 European countries. Int J Cancer 2010;126:692-702.

34. Shields M, Connor Gorber S, Tremblay MS. Estimates of obesity based on self-report versus direct measures. Health Rep 2008;19:61-76.

35. Momenimovahed Z, Ghoncheh M, Pakzad R, Hasanpour $H$, Salehiniya $H$. Incidence and mortality of uterine cancer and relationship with human development index in the world. Cukurova Med J 2017;42:233-40. 